\title{
Research Article \\ Design of Nonuniform Filter Bank Transceivers for Frequency Selective Channels
}

\author{
Han-Ting Chiang, ${ }^{1}$ See-May Phoong, ${ }^{1}$ and Yuan-Pei Lin ${ }^{2}$ \\ ${ }^{1}$ Department of Electrical Engineering, Graduate Institute of Communication Engineering, National Taiwan University, \\ Taipei 10617, Taiwan \\ ${ }^{2}$ Department of Electrical and Control Engineering, National Chiao Tung University, Hsinchu 300, Taiwan
}

Received 14 January 2006; Revised 16 July 2006; Accepted 13 August 2006

Recommended by Soontorn Oraintara

\begin{abstract}
In recent years, there has been considerable interest in the theory and design of filter bank transceivers due to their superior frequency response. In many applications, it is desired to have transceivers that can support multiple services with different incoming data rates and different quality-of-service requirements. To meet these requirements, we can either do resource allocation or design transceivers with a nonuniform bandwidth partition. In this paper, we propose a method for the design of nonuniform filter bank transceivers for frequency selective channels. Both frequency response and signal-to-interference ratio (SIR) can be incorporated in the transceiver design. Moreover, the technique can be extended to the case of nonuniform filter bank transceivers with rational sampling factors. Simulation results show that nonuniform filter bank transceivers with good filter responses as well as high SIR can be obtained by the proposed design method.
\end{abstract}

Copyright ( 2007 Hindawi Publishing Corporation. All rights reserved.

\section{INTRODUCTION}

The orthogonal frequency division multiplexing (OFDM) system has enjoyed great success in many wideband communication systems due to its ability to combat intersymbol interference (ISI) [1]. It is known that the transmitting and receiving filters of the OFDM transceiver have poor frequency responses. As a result, many subchannels will be affected when there is narrowband interference, and the performance degrades significantly [2]. Many techniques have been proposed to solve this problem.

One of the solutions is the filter bank technique. In recent years, there has been considerable interest in the application of filter banks to the design of transceivers with good frequency characteristics [2-10]. Many of these previous studies [3-6] have focused on the design of filter bank transceivers (or transmultiplexers) under the assumption that the transmission channel is an ideal channel that does not create ISI. When the channel is a frequency selective channel, these filter bank transceivers suffer from severe ISI effect $[7,8]$, and post processing technique is needed at the receiver for channel equalization [4]. Recently the authors in [10] studied the filter bank transceiver for frequency selective channels. The transmitting and receiving filters are optimized for SIR (signal-to-interference ratio) maximization. Like OFDM systems, simple one-tap equalizers can be employed at the receiver for channel equalization. It has been demonstrated that filter bank transceivers with high SIR and good frequency responses can be obtained [10].

In many applications, it is desired to have transceivers that can support multiple services [11, 12]. Different services might have different incoming data rates and different quality-of-service requirements. One solution to this problem is to judiciously allocating the resources to meet the requirements, see, for example, [11]. Another solution is to use a nonuniform filter bank transceiver. The theory and design of nonuniform filter banks have been studied by a number of researchers [13-18]. These results are extended to the design of transceivers and transmultiplexers with nonuniform band separation in $[12,19]$. In [12], the authors proposed a general building block for the design of nonuniform filter bank transmultiplexers. Near perfect reconstruction transmultiplexers with good frequency property can be obtained by the proposed method therein. In [19], a design of nonuniform transmultiplexers using semi-infinite programming was proposed. The proposed algorithm was efficient and good results were achieved. However these nonuniform transceiver designs do not consider the channel effect. When 


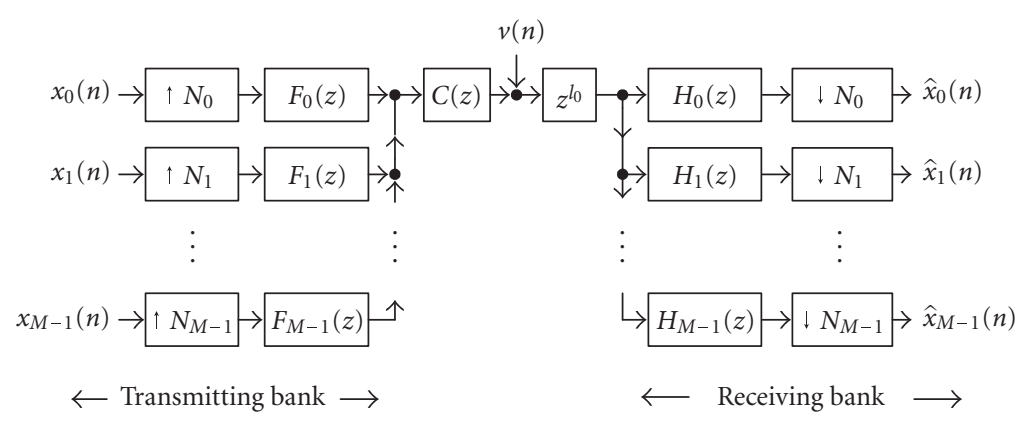

FIGURE 1: A nonuniform filter bank transceiver with integer sampling factors.

the transmission channel is frequency selective, an additional equalizer is needed at the receiver.

In this paper, we consider the design of nonuniform transceiver for frequency selective channels. Both the cases of integer and rational sampling factors are considered. As the effect of channel is taken into consideration at the time the filter bank is optimized, simple one-tap equalizers can be used at the receiver for channel equalization. Unlike the uniform case, the equivalent system from the transmitter input to the receiver output is no longer LTI and ISI-free condition needs to be derived. Furthermore we will show that like the uniform case [10], SIR can be formulated as a Rayleigh-Ritz ratio of filter coefficients. The optimal filters that maximize the SIR can be obtained from an eigenvector of a positive definite matrix. Moreover, an iterative algorithm that can incorporate the frequency response is proposed for SIR maximization. Simulation results show that we can obtain nonuniform transceivers with very high SIR (around $50 \mathrm{~dB}$ ) and good frequency response (stopband attenuation around $40 \mathrm{~dB}$ ).

This paper is organized as follows. In Section 2, we study nonuniform filter bank transceivers with integer sampling factors. The ISI-free condition is derived and the SIR is formulated as a Rayleigh-Ritz ratio of transmitting and receiving filters. Then SIR-optimized transmitting and receiving filters are given. Moreover, the design method can be extended to the case of unknown frequency selective channels. In Section 3, an iterative algorithm is proposed to alternatingly optimize the transmitting and receiving filters for SIR maximization. We will show how to incorporate the frequency response into the objective function. The results are extended to the case of rational sampling factor in Section 4. In Section 5, simulation examples are given to demonstrate the usefulness of the proposed method. A conclusion is given in Section 6.

\section{Notation}

The $N$-fold downsampled and upsampled versions of $x(n)$ are respectively denoted by $[x(n)]_{\downarrow N}$ and $[x(n)]_{\uparrow N}$ in the time domain, and by $[X(z)]_{\downarrow N}$ and $[X(z)]_{\uparrow N}$ in the $z$ domain. The convolution of two sequences $x(n)$ and $y(n)$ is represented by $x(n) * y(n)$.

\section{NONUNIFORM FILTER BANK TRANSCEIVERS WITH INTEGER SAMPLING FACTORS}

Figure 1 shows a nonuniform filter bank transceiver. The downsampling and upsampling ratios $N_{i}$ are integers and they can be different for different $i$. A larger $N_{i}$ indicates a lower data rate and also implies that a smaller bandwidth is allocated to the $i$ th subband. For a filter bank transceiver, the integers $N_{i}$ satisfy $\sum_{i=0}^{M-1} 1 / N_{i} \leq 1$, which is a necessary condition for recovering the input signals $x_{i}(n)$. When the equality $\sum_{i=0}^{M-1} 1 / N_{i}=1$ holds, the transceiver is said to be critically sampled. The transmission channel is modeled as an $L$ th-order LTI channel with transfer function

$$
C(z)=\sum_{l=0}^{L} c(l) z^{-l}
$$

The additive noise is denoted by $v(n)$. Because our formulation is based on the signal-to-interference ratio, the channel noise does not affect the transceiver design. Therefore in Sections 2, 3, and 4, we set $v(n)=0$. For convenience, an advance operator $z^{l_{0}}$ is added at the receiver to account for the system delay caused by channel $C(z)$. In practice, this advance element can be replaced by an appropriate delay. In this paper, we consider only FIR filter banks. The transmitting and receiving filters are, respectively,

$$
F_{i}(z)=\sum_{n=0}^{N_{f_{i}}} f_{i}(n) z^{-n}, \quad H_{i}(z)=\sum_{n=0}^{N_{h_{i}}} h_{i}(n) z^{n} .
$$

The orders of these filters $N_{f_{i}}$ and $N_{h_{i}}$ can be larger than $N_{i}$. For notational simplicity, we use the noncausal expression for the receiving filters. Causal filters can be obtained easily by adding sufficient delays. In addition, we assume that the input signals $x_{i}(n)$ are uncorrelated, zero mean, wide sense stationary (WSS), and white random processes with the same variance $\varepsilon_{x}$. That is,

$$
E\left[x_{i}(n)\right]=0, \quad E\left[x_{i}(n) x_{j}^{*}(m)\right]=\mathcal{E}_{x} \delta(i-j) \delta(n-m) .
$$

This assumption is usually satisfied by properly interleaving the input data. 


\subsection{ISI-free condition}

The filter bank transceiver shown in Figure 1 is said to be ISIfree if in the absence of noise, for all possible input signals $x_{i}(n)$, the outputs are

$$
\hat{x}_{i}(n)=G_{i} x_{i}(n),
$$

for some constant $G_{i}$. In this case, a zero-forcing solution can be obtained by cascading a simple one-tap equalizer. Expressing the output signal at the $j$ th subband in the $z$ domain, we have

$$
\begin{aligned}
\hat{X}_{j}(z)= & \sum_{i=0}^{M-1}\left[X_{i}\left(z^{N_{i}}\right) F_{i}(z) z^{l_{0}} C(z) H_{j}(z)\right]_{\downarrow N_{j}} \\
= & X_{j}(z)\left[F_{j}(z) z^{l_{0}} C(z) H_{j}(z)\right]_{N_{j}} \\
& +\sum_{\substack{i=0 \\
i \neq j}}^{M-1}\left[X_{i}\left(z^{N_{i}}\right) F_{i}(z) z^{l_{0}} C(z) H_{j}(z)\right]_{\downarrow_{j}} .
\end{aligned}
$$

From the above equation, we see that in general the system from the input $x_{i}(n)$ to the output $\hat{x}_{j}(n)$ is not LTI unless $N_{j}$ is a factor of $N_{i}$. This is very different from the case of uniform filter bank transceivers, in which all $N_{i}=N$. Let $g_{i, j}$ be the greatest common divisor (gcd) of $N_{i}$ and $N_{j}$. Define two coprime integers $p_{i, j}=N_{i} / g_{i, j}$ and $p_{j, i}=N_{j} / g_{i, j}$. Then we can write

$$
\begin{aligned}
\hat{X}_{j}(z)= & X_{j}(z)\left[F_{j}(z) z^{l_{0}} C(z) H_{j}(z)\right]_{\downarrow N_{j}} \\
& +\sum_{\substack{i=0 \\
i \neq j}}^{M-1}\left[X_{i}\left(z^{p_{i, j}}\right)\left[F_{i}(z) z^{l_{0}} C(z) H_{j}(z)\right]_{\downarrow g_{i, j}}\right]_{\downarrow p_{j, i}} .
\end{aligned}
$$

Define

$$
\begin{aligned}
T_{i, j}(z) & =\left[F_{i}(z) z^{l_{0}} C(z) H_{j}(z)\right]_{\downarrow g_{i j}} \\
& =\sum_{l=0}^{L} c(l)\left[F_{i}(z) H_{j}(z) z^{l_{0}-l}\right]_{\downarrow g_{i, j}}
\end{aligned}
$$

for $0 \leq i, j \leq M-1$. As the input signals $x_{i}(n)$ are arbitrary, one can show (see the appendix for a proof) that the ISI-free condition $\hat{X}_{i}(z)=G_{i} X_{i}(z)$ is satisfied if and only if

$$
T_{i, j}(z)= \begin{cases}G_{i}, & j=i, \\ 0, & \text { otherwise. }\end{cases}
$$

For convenience of discussion, we express $\left[F_{i}(z) H_{j}(z) z^{l_{0}-l}\right]_{l g_{i, j}}$ in terms of the two sequences $\alpha_{i, l}(n)$ and $\beta_{i, j, l}(n)$ as

$$
\left[F_{i}(z) H_{j}(z) z^{l_{0}-l}\right]_{\mid g_{i, j}}= \begin{cases}\alpha_{i, l}(0)+\sum_{\substack{n \\ n \neq 0}} \alpha_{i, l}(n) z^{-n}, & i=j, \\ \sum_{n} \beta_{i, j, l}(n) z^{-n}, & i \neq j,\end{cases}
$$

for $0 \leq i, j \leq M-1$, and $0 \leq l \leq L$. Note that since $F_{i}(z)$ and $H_{j}(z)$ are of finite length, $\alpha_{i, l}(n)$ and $\beta_{i, j, l}(n)$ have finite nonzero terms only. Using the above definition, we can write the $j$ th output signal $\hat{x}_{j}(n)$ as

$$
\begin{aligned}
\hat{x}_{j}(n)= & {\left[\sum_{l=0}^{L} \alpha_{j, l}(0) c(l)\right] x_{j}(n) } \\
& +\sum_{l=0}^{L} c(l)\left[\alpha_{j, l}(n)-\alpha_{j, l}(0) \delta(n)\right] * x_{j}(n) \\
& +\sum_{\substack{i=0 \\
i \neq j}}^{M-1}\left[\sum_{l=0}^{L} c(l) \beta_{i, j, l}(n) *\left[x_{i}(n)\right]_{\uparrow p_{i, j}}\right]_{\downarrow p_{j, i}} .
\end{aligned}
$$

The first, second, and third terms on the right-hand side of the above expression are the desired signal, the intraband ISI and the cross-band ISI, respectively. To get an ISI-free transceiver, we need to find the transmitting filters $F_{k}(z)$ and receiving filters $H_{k}(z)$ so that the second and third terms are equal to zero. The general solution to this problem is still unknown. In the following, we will show how to reduce the effect of ISI by finding a solution that maximizes the signalto-interference ratio (SIR).

\subsection{Matrix formulations of $\alpha_{i, l}(n)$ and $\beta_{i, j, l}(n)$}

In this section, we will formulate the sequences $\alpha_{i, l}(n)$ and $\beta_{i, j, l}(n)$ in a matrix form. These expressions will be useful for the optimization of the transceivers. Recall from (9) that $\alpha_{i, l}(n)$ and $\beta_{i, j, l}(n)$ are obtained from the convolution of $f_{k}(n)$ and $h_{k}(n)$. Let us define the following vectors:

$$
\begin{array}{cc}
\boldsymbol{\alpha}_{i}(n)=\left[\begin{array}{c}
\alpha_{i, 0}(n) \\
\alpha_{i, 1}(n) \\
\vdots \\
\alpha_{i, L}(n)
\end{array}\right], \quad \boldsymbol{\beta}_{i, j}(n)=\left[\begin{array}{c}
\beta_{i, j, 0}(n) \\
\beta_{i, j, 1}(n) \\
\vdots \\
\beta_{i, j, L}(n)
\end{array}\right], \\
\mathbf{h}_{i}=\left[\begin{array}{c}
h_{i}(0) \\
h_{i}(1) \\
\vdots \\
h_{i}\left(N_{h_{i}}\right)
\end{array}\right], \quad \mathbf{f}_{i}=\left[\begin{array}{c}
f_{i}(0) \\
f_{i}(1) \\
\vdots \\
f_{i}\left(N_{f_{i}}\right)
\end{array}\right] .
\end{array}
$$

Then from (9), it is not difficult to verify that the vectors $\boldsymbol{\alpha}_{i}(n)$ and $\boldsymbol{\beta}_{i, j}(n)$ can respectively be expressed as

$$
\begin{gathered}
\boldsymbol{\alpha}_{i}(n)=\mathbf{A}_{i}(n) \mathbf{h}_{i}, \\
\boldsymbol{\beta}_{i, j}(n)=\mathbf{B}_{i, j}(n) \mathbf{h}_{j},
\end{gathered}
$$


where the matrices $\mathbf{A}_{i}(n)$ and $\mathbf{B}_{i, j}(n)$ are respectively given by

$\mathbf{A}_{i}(n)$

$$
=\left[\begin{array}{cccc}
f_{i}\left(n N_{i}+l_{0}\right) & f_{i}\left(n N_{i}+l_{0}+1\right) & \cdots & f_{i}\left(n N_{i}+l_{0}+N_{h_{i}}\right) \\
f_{i}\left(n N_{i}+l_{0}-1\right) & f_{i}\left(n N_{i}+l_{0}-1+1\right) & \cdots & f_{i}\left(n N_{i}+l_{0}-1+N_{h_{i}}\right) \\
\vdots & \vdots & \ddots & \vdots \\
f_{i}\left(n N_{i}+l_{0}-L\right) & f_{i}\left(n N_{i}+l_{0}-L+1\right) & \cdots & f_{i}\left(n N_{i}+l_{0}-L+N_{h_{i}}\right)
\end{array}\right] \text {, }
$$

$\mathbf{B}_{i, j}(n)$

$$
=\left[\begin{array}{cccc}
f_{i}\left(n g_{i, j}+l_{0}\right) & f_{i}\left(n g_{i, j}+l_{0}+1\right) & \cdots & f_{i}\left(n g_{i, j}+l_{0}+N_{h_{j}}\right) \\
f_{i}\left(n g_{i, j}+l_{0}-1\right) & f_{i}\left(n g_{i, j}+l_{0}-1+1\right) & \cdots & f_{i}\left(n g_{i, j}+l_{0}-1+N_{h_{j}}\right) \\
\vdots & \vdots & \ddots & \vdots \\
f_{i}\left(n g_{i, j}+l_{0}-L\right) & f_{i}\left(n g_{i, j}+l_{0}-L+1\right) & \cdots & f_{i}\left(n g_{i, j}+l_{0}-L+N_{h_{j}}\right)
\end{array}\right] \text {. }
$$

The dimensions of the matrices $\mathbf{A}_{i}(z)$ and $\mathbf{B}_{i, j}(n)$ are, respectively, $(L+1) \times\left(N_{h_{i}}+1\right)$ and $(L+1) \times\left(N_{h_{j}}+1\right)$. Notice that $g_{i, j}=N_{i}$ when $i=j$. Similarly, we can also express the vectors $\boldsymbol{\alpha}_{i}(n)$ and $\boldsymbol{\beta}_{i, j}(n)$, respectively, in terms of the transmitting filter $\mathbf{f}_{i}$ as

$$
\boldsymbol{\alpha}_{i}(n)=\widetilde{\mathbf{A}}_{i}(n) \mathbf{f}_{i}, \quad \boldsymbol{\beta}_{i, j}(n)=\widetilde{\mathbf{B}}_{i, j}(n) \mathbf{f}_{i},
$$

for some matrices $\widetilde{\mathbf{A}}_{i}(n)$ and $\widetilde{\mathbf{B}}_{i, j}(n)$. The matrices $\widetilde{\mathbf{A}}_{i}(n)$ and $\widetilde{\mathbf{B}}_{i, j}(n)$ consist of the transmitting filter coefficients $h_{j}(n)$ and they are very similar to $\mathbf{A}_{i}(n)$ and $\mathbf{B}_{i, j}(n)$, respectively.

\subsection{SIR-optimized receiving filters}

In this section, we will design the receiving filters so that the SIR is maximized for a fixed set of transmitting filters. As the $j$ th receiving filter affects only the $j$ th output signal $\hat{x}_{j}(n)$, the receiving filters can be designed separately; the $j$ th receiving filter $F_{j}(z)$ is optimized so that the SIR of the $j$ th output signal $\hat{x}_{j}(n)$ is maximized. Recall from (10) that the output of the $j$ th subband $\hat{x}_{j}(n)$ consists of three components, namely, the desired signal, the intraband interference, and the cross-band interference. As the input signals $x_{i}(n)$ satisfy the uncorrelated and white property in (3), the desired signal power and intraband interference power at the $j$ th output are given by

$$
\begin{gathered}
P_{\text {sig }}(j)=\mathcal{E}_{x}\left|\sum_{l=0}^{L} \alpha_{j, l}(0) c(l)\right|^{2}, \\
P_{\text {intra }}(j)=\mathcal{E}_{x} \sum_{n, n \neq 0}\left|\sum_{l=0}^{L} \alpha_{j, l}(n) c(l)\right|^{2},
\end{gathered}
$$

where $\varepsilon_{x}$ is the power of the input signal defined in (3). The computation of the cross-band interference power is more complicated because the sequence $\left[x_{j}(n)\right]_{\uparrow_{i, j}}$ is not a WSS process. From multirate theory $[20]$, we know that $\left[x_{j}(n)\right]_{i p_{i, j}}$ is cyclo wide sense stationary with period $p_{i, j}, \operatorname{or} \operatorname{CWSS}\left(p_{i, j}\right)$. Letting $u(n)=\left[x_{j}(n)\right]_{\uparrow_{i, j}}$, then its autocorrelation coefficients satisfy $E\left[u(n) u^{*}(n-k)\right]=E\left[u\left(n+p_{i, j}\right) u^{*}\left(n+p_{i, j}-k\right)\right]$. Since $p_{i, j}$ and $p_{j, i}$ are coprime, the quantity

$$
\left[\sum_{l=0}^{L} c(l) \beta_{i, j, l}(n) *\left[x_{i}(n)\right]_{\uparrow p_{i, j}}\right]_{\downarrow p_{j, i}}
$$

is also CWSS $\left(p_{i, j}\right)[20]$. From (10), we see that the crossband interference consists of $(M-1)$ CWSS sequences with period $p_{i, j}$ for $i=0, \ldots, j-1, j+1, \ldots, M-1$. Let $P_{j}$ be the least common multiple of the integers $\left\{p_{0, j}, \ldots\right.$, $\left.p_{j-1, j}, p_{j+1, j}, \ldots, p_{M-1, j}\right\}$. Then the cross-band interference is a CWSS $\left(P_{j}\right)$ random process. We can compute the average cross-band interference power over one period $P_{j}$ and it is given by

$$
P_{\text {cross }}(j)=\mathcal{E}_{x} \sum_{\substack{i, n \\ i \neq j}} \frac{1}{p_{i, j}}\left|\sum_{l=0}^{L} \beta_{i, j, l}(n) c(l)\right|^{2} .
$$

Next we will express the three quantities $P_{\text {sig }}(j), P_{\text {intra }}(j)$, and $P_{\text {cross }}(j)$ in terms of the receiving filter coefficients $h_{j}(n)$. To do this, let us define the $(L+1) \times 1$ vector

$$
\mathbf{c}=\left[\begin{array}{llll}
c(0) & c(1) & \cdots & c(L)
\end{array}\right]^{T} .
$$

Then from (12), we can write

$$
\begin{aligned}
\mathcal{E}_{x}\left|\sum_{l=0}^{L} \alpha_{j, l}(0) c(l)\right|^{2} & =\varepsilon_{x}\left\|\mathbf{c}^{T} \mathbf{A}_{j}(0) \mathbf{h}_{j}\right\|^{2} \\
& =\varepsilon_{x} \mathbf{h}_{j}^{\dagger} \mathbf{A}_{j}^{\dagger}(0) \mathbf{c}^{*} \mathbf{c}^{T} \mathbf{A}_{j}(0) \mathbf{h}_{j} .
\end{aligned}
$$

Similarly, using the expressions of $\boldsymbol{\alpha}_{i}(n)$ and $\boldsymbol{\beta}_{i, j}(n)$ in (12), we can also write the intraband and cross-band interference powers in a quadratic form of $\mathbf{h}_{j}$. In summary, the three powers are given by

$$
\begin{gathered}
P_{\text {sig }}(j)=\mathbf{h}_{j}^{\dagger} \mathbf{Q}_{\text {sig }, j} \mathbf{h}_{j}, \quad P_{\text {intra }}(j)=\mathbf{h}_{j}^{\dagger} \mathbf{Q}_{\text {intra }, j} \mathbf{h}_{j}, \\
P_{\text {cross }}(j)=\mathbf{h}_{j}^{\dagger} \mathbf{Q}_{\text {cross }, j} \mathbf{h}_{j},
\end{gathered}
$$

where the matrices $\mathbf{Q}_{\mathrm{sig}, j}, \mathbf{Q}_{\mathrm{intra}, j}$, and $\mathbf{Q}_{\mathrm{cross}, j}$ are, respectively, given by

$$
\begin{gathered}
\mathbf{Q}_{\mathrm{sig}, j}=\mathcal{E}_{x} \mathbf{A}_{j}^{\dagger}(0) \mathbf{c}^{*} \mathbf{c}^{T} \mathbf{A}_{j}(0), \\
\mathbf{Q}_{\text {intra }, j}=\mathcal{E}_{x} \sum_{n, n \neq 0} \mathbf{A}_{j}^{\dagger}(n) \mathbf{c}^{*} \mathbf{c}^{T} \mathbf{A}_{j}(n), \\
\mathbf{Q}_{\mathrm{cross}, j}=\boldsymbol{E}_{x} \sum_{\substack{i, n \\
i \neq j}} \frac{1}{p_{i, j}} \mathbf{B}_{i, j}^{\dagger}(n) \mathbf{c}^{*} \mathbf{c}^{T} \mathbf{B}_{i, j}(n) .
\end{gathered}
$$

As $x_{i}(n)$ and $x_{j}(n)$ are uncorrelated for $i \neq j$, the total ISI power at the $j$ th output is $P_{\text {isi }}(j)=P_{\text {intra }}(j)+P_{\text {cross }}(j)$. Thus 
the SIR of the $j$ th output is given by

$$
\gamma_{j}=\frac{P_{\text {sig }}(j)}{P_{\text {isi }}(j)}=\frac{\mathbf{h}_{j}^{\dagger} \mathbf{Q}_{\text {sig }, j} \mathbf{h}_{j}}{\mathbf{h}_{j}^{\dagger} \mathbf{Q}_{\mathrm{isi}, j} \mathbf{h}_{j}},
$$

where $\mathbf{Q}_{\mathrm{isi}, j}=\mathbf{Q}_{\text {intra, } j}+\mathbf{Q}_{\text {cross, } j \text {. Notice that both } \mathbf{Q}_{\text {sig }, j} \text { and }}$ $\mathbf{Q}_{\text {isi,j }}$ are positive semidefinite matrices. Furthermore, except for some very rare cases, the matrix $\mathbf{Q}_{\text {isi,j }}$ is positive definite. From the above expression, we see that the SIR is expressed as a Rayleigh-Ritz ratio of $\mathbf{h}_{j}$. The optimal unit-norm vector $\mathbf{h}_{j}$ that maximizes $\gamma_{j}$ is well known [21]. Let $\mathbf{Q}_{i s i, j}^{1 / 2}$ be the positive definite matrix such that $\mathbf{Q}_{\mathrm{isi}, j}=\mathbf{Q}_{\mathrm{isi}, j}^{1 / 2} \mathbf{Q}_{\mathrm{isi}, j}^{1 / 2}$. The optimal $\mathbf{h}_{j}$ is given by

$$
\mathbf{h}_{j, \mathrm{opt}}=\mathbf{Q}_{\mathrm{isi}, j}^{-1 / 2} \arg \max _{\mathbf{v} \neq \mathbf{0}} \frac{\mathbf{v}^{\dagger} \mathbf{Q}_{\mathrm{isi}, j}^{-1 / 2} \mathbf{Q}_{\mathrm{sig}, j} \mathbf{Q}_{\mathrm{isi}, j}^{-1 / 2} \mathbf{v}}{\mathbf{v}^{\dagger} \mathbf{v}}
$$

The optimal vector $\mathbf{v}$ is the eigenvector corresponding to the largest eigenvalue of the positive definite matrix $\mathbf{Q}_{\mathrm{isi}, j}^{-1 / 2} \mathbf{Q}_{\mathrm{sig}, j} \mathbf{Q}_{\mathrm{isi}, j}^{-1 / 2}$.

\subsection{SIR-optimized transmitting filters}

In this section, we consider the SIR optimization of the transmitting filters $f_{i}(n)$ given a fixed set of the receiving filters. As the $i$ th transmitting filter $f_{i}(n)$ affects only the $i$ th input signal $x_{i}(n)$, we can consider the SIR due to the $i$ th transmitted signal $x_{i}(n)$. Consider the transmission scenario when only the $i$ th subband is transmitting, that is, $x_{j}(n)=0$ for $j \neq i$. Then from (10), the outputs of the receiver are given by

$$
\begin{gathered}
\hat{x}_{i}(n)=\left[\sum_{l=0}^{L} \alpha_{i, l}(0) c(l)\right] x_{i}(n) \\
+\sum_{l=0}^{L} c(l)\left[\alpha_{i, l}(n)-\alpha_{i, l}(0) \delta(n)\right] * x_{i}(n), \\
\hat{x}_{j}(n)=\left[\sum_{l=0}^{L} c(l) \beta_{i, j, l}(n) *\left[x_{i}(n)\right]_{\uparrow p_{i, j}}\right]_{\downarrow p_{j, i}}, \quad \text { for } i \neq j .
\end{gathered}
$$

Note that the first and second terms on the right-hand side of (24) are respectively the desired signal and the intraband interference due to the $i$ th transmitted signal $x_{i}(n)$. On the other hand, $\hat{x}_{j}(n)$ represents the cross-band interferences due to $x_{i}(n)$. By following a procedure similar to that in the previous section, we can compute the signal power and interference powers and express the SIR as a Rayleigh-Ritz ratio as follows:

$$
\tilde{\gamma}_{i}=\frac{\mathbf{f}_{i}^{\dagger} \widetilde{\mathbf{Q}}_{\mathrm{sig}, i} \mathbf{f}_{i}}{\mathbf{f}_{i}^{\dagger} \widetilde{\mathbf{Q}}_{\mathrm{isi}, i} \mathbf{f}_{i}}
$$

where the matrices $\widetilde{\mathbf{Q}}_{\text {sig }, i}$ and $\widetilde{\mathbf{Q}}_{\text {isi, } i \text { are positive semidefinite }}$ matrices that have a form very similar to $\mathbf{Q}_{\mathrm{sig}, i}$ and $\mathbf{Q}_{\mathrm{isi}, i}$, respectively. Hence the optimal unit-norm $\mathbf{f}_{i}$ that maximizes the SIR can be obtained by solving the above Rayleigh-Ritz ratio.

\subsection{SIR optimized for unknown channels}

In many applications, the exact channel impulse response may not be available, and we may have only the statistics of the transmission channels. The above design method can easily be modified to obtain transceivers that are optimized for unknown channels. Assume that the vector containing the channel impulse response, $\mathbf{c}$, is zero-mean with autocorrelation matrix

$$
\mathbf{R}_{c}=E\left[\mathbf{c c}^{\dagger}\right]
$$

In this case, the exact channel impulse response is not known. From previous discussions, we know that the signal power and interference powers at the output of the $j$ th subband are respectively given by (20) and (21). When the channel is not known, we can compute the average signal power and interference powers by taking the expectation with respect to the channel impulse response $c(l)$. It is not difficult to verify that the average SIR can also be expressed as a Rayleigh-Ritz ratio of the filter coefficients $\mathbf{h}_{i}$.

Similarly, given the receiving filters, we can modify the optimization of transmitting filters $\mathbf{f}_{i}$ for the case of unknown channels by using the average SIR. In many situations, we do not know the statistics of the channel. In this case, it is often assumed that the channel impulse responses are independent identical distribution, that is, i.i.d. channels. The autocorrelation matrix of the channel impulse response becomes $\mathbf{R}_{c}=\sigma_{c}^{2} \mathbf{I}$.

\section{AN ITERATIVE ALGORITHM FOR SIR OPTIMIZATION WITH FREQUENCY CRITERIA}

From the previous discussions, we know that when the transmitting filters are given, we can obtain optimum receiving filters so that SIR is maximized. Conversely, given the receiving filters we can design the transmitting filters that maximize the SIR. One can therefore alternatingly optimize the receiving and transmitting filters so that SIR is maximized. Because in each iteration, the solution obtained in the previous iteration is also a candidate, the SIR cannot decrease ${ }^{1}$ when the number of iterations increases. As we will see in the numerical examples, the increase in SIR is substantial as the number of iterations increases. However because no constraint is applied on the filters, their frequency responses will often degrade significantly as the number of iterations increases. To solve this problem, we can incorporate the filter stopband energy in the optimization. Let us consider the design of the receiving filters $\mathbf{h}_{j}$. The stopband energy of the $j$ th receiving filter $H_{j}(z)$ is given by

$$
P_{\text {stop }}(j)=\frac{1}{2 \pi} \int_{\mathfrak{R}_{h, j}}\left|H_{j}\left(e^{j \omega}\right)\right|^{2} d \omega,
$$

where $\mathfrak{R}_{h, j}$ is the stopband region of $H_{j}(z)$. Define the vector $\mathbf{e}_{N}(z)=\left[\begin{array}{llll}1 & z & \cdots & z^{N}\end{array}\right]^{T}$. Then the weighted stopband

\footnotetext{
${ }^{1}$ In general, it is not guaranteed that the SIR is monotonically increasing.
} 


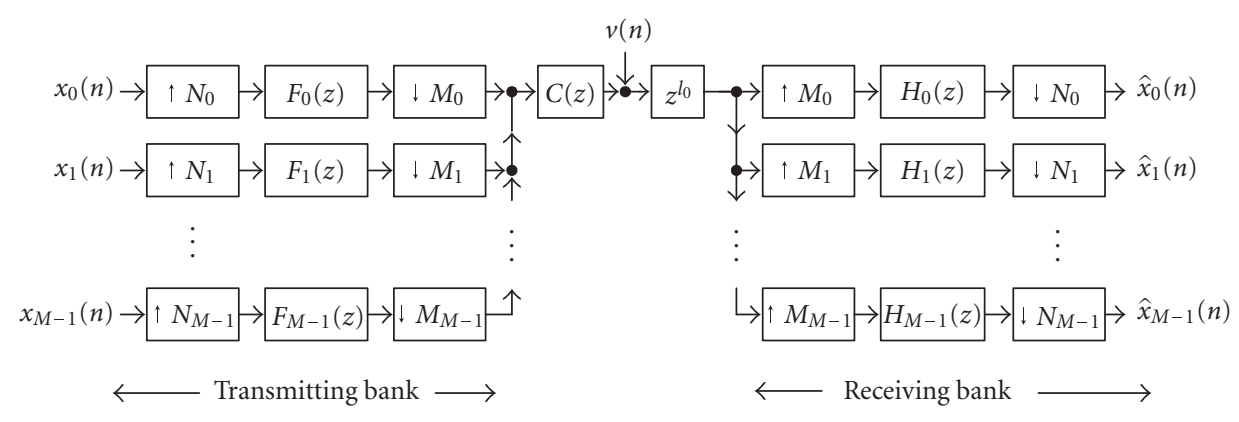

FIgURE 2: Nonuniform filter bank transceiver with rational sampling factors.

energy can be expressed as

$$
\mathbf{P}_{\text {stop }}(j)=\mathbf{h}_{j}^{\dagger} \mathbf{Q}_{\text {stop }, j} \mathbf{h}_{j}
$$

where the matrix $\mathbf{Q}_{\text {stop, } j}$ is given by

$$
\mathbf{Q}_{\text {stop }, j}=\frac{1}{2 \pi} \int_{\mathfrak{R}_{h, j}} \mathbf{e}_{N_{h_{j}}}\left(e^{j \omega}\right) \mathbf{e}_{N_{h_{j}}}^{\dagger}\left(e^{j \omega}\right) d \omega .
$$

The new objective function that incorporates the frequency response is

$$
\eta_{j}=\frac{\mathbf{h}_{j}^{\dagger} \mathbf{Q}_{\mathrm{sig}, j} \mathbf{h}_{j}}{\mathbf{h}_{j}^{\dagger}\left[\mathbf{Q}_{\mathrm{isi}, j}+c_{h, j} \mathbf{Q}_{\mathrm{stop}, j}\right] \mathbf{h}_{j}},
$$

where $c_{h, j} \geq 0$ is a weight that adjusts the relative importance of the frequency responses. When $c_{h, j}=0$, the new objective function $\eta_{j}$ reduces to the SIR expression $\gamma_{j}$ in (22) and no frequency criteria are applied. One can see that $\eta_{j}$ is also a Rayleigh-Ritz ratio of $\mathbf{h}_{j}$. We can choose $\mathbf{h}_{j}$ to be the unitnorm vector that maximizes this ratio. Similarly, one can incorporate the stopband energy into the optimization of the transmitting filters $f_{i}(n)$. One will get a new objective function

$$
\tilde{\eta}_{i}=\frac{\mathbf{f}_{i}^{\dagger} \widetilde{\mathbf{Q}}_{\text {sig }, i} \mathbf{f}_{i}}{\mathbf{f}_{i}^{\dagger}\left[\widetilde{\mathbf{Q}}_{\mathrm{isi}, i}+c_{f, i} \widetilde{\mathbf{Q}}_{\text {stop }, i}\right] \mathbf{f}_{i}},
$$

where $\mathbf{f}_{i}^{\dagger} \widetilde{\mathbf{Q}}_{\text {stop }, i} \mathbf{f}_{i}$ is the term corresponding to the stopband energy of the filter $f_{i}(n)$. The optimal $\mathbf{f}_{i}$ is the unit-norm vector that maximizes $\tilde{\eta}_{i}$.

Note that in the new objective function, the passband responses of the filters are not included. For unit-norm filters, when the stopband energy is small, the passband energy will be close to one. In transceiver designs, nearly zero ISI property can be guaranteed by a high SIR and the flatness of passband response is not needed.

The iterative algorithm for transceiver optimization is summarized as follows.

(1) Select a set of the receiving filters $H_{i}^{(0)}(z)$ with good frequency responses.
For $k \geq 1$, repeat the following steps.

(2) Given the receiving filters $H_{i}^{(k-1)}(z)$, optimize $F_{j}^{(k)}(z)$ so that $\tilde{\eta}_{j}$ is maximized for $0 \leq j \leq M-1$.

(3) Given the transmitting filter $F_{j}^{(k)}(z)$, optimize $H_{i}^{(k)}(z)$ so that $\eta_{i}$ is maximized for $0 \leq i \leq M-1$.

(4) Stop if the SIR is higher than the desired value or if it reaches the maximum number of iterations; otherwise, $k=k+1$ and return to step (2).

\section{NONUNIFORM FILTER BANK TRANSCEIVERS WITH RATIONAL SAMPLING FACTORS}

In this section, we generalize the design method to the case of rational sampling factors. We will first employ the technique in [15] to convert the transceiver with rational sampling factors into an equivalent transceiver with integer sampling factor. Then the optimization method developed in the previous sections can be adopted. The block diagram of a nonuniform filter bank transceiver with rational sampling factors is shown in Figure 2. At the transmitter, the input signal $x_{i}(n)$ goes through an $N_{i}$-fold expander and an $M_{i}$-fold decimator. The bandwidth of the $i$ th subband is proportional to the ratio $M_{i} / N_{i}$. Without loss of generality, we assume that the integers $M_{i}$ and $N_{i}$ are coprime. If they are not coprime, then it is known [20] that the $i$ th subband can be replaced with an equivalent system with coprime $M_{i}^{\prime}$ and $N_{i}^{\prime}$, and such an equivalent system will have a lower complexity. Furthermore, to ensure symbol recovery, we assume

$$
\sum_{i=0}^{M-1} \frac{M_{i}}{N_{i}} \leq 1
$$

Let us decompose the $k$ th transmitting and receiving filters using the polyphase representation as

$$
\begin{aligned}
& H_{k}(z)=\sum_{\ell=0}^{M_{k}-1} z^{\ell} E_{k, \ell}\left(z^{M_{k}}\right), \\
& F_{k}(z)=\sum_{\ell=0}^{M_{k}-1} z^{-\ell} R_{k, \ell}\left(z^{M_{k}}\right) .
\end{aligned}
$$

Note that no coefficient of $H_{k}(z)$ or $F_{k}(z)$ appears in more 


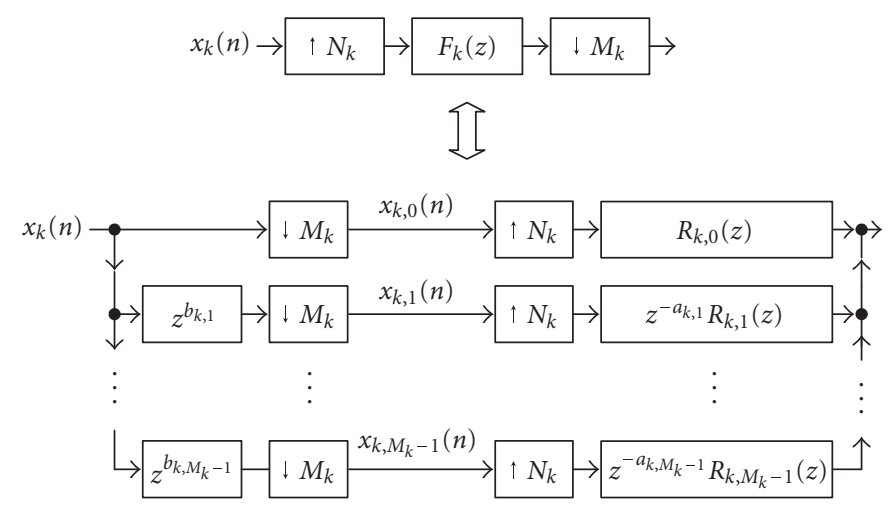

(a)

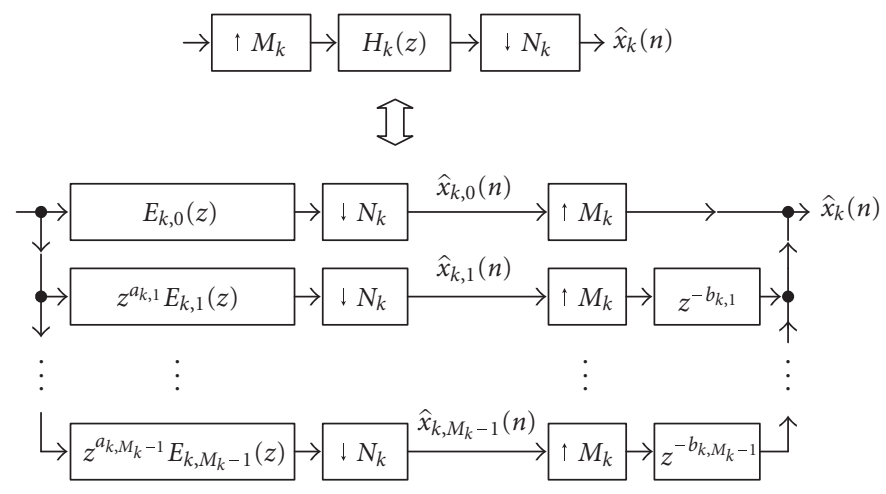

(b)

FIGURE 3: (a) Equivalent circuit of the $k$ th subband in the transmitting bank, (b) equivalent circuit of the $k$ th subband in the receiving bank.

than one $E_{k, \ell}(z)$ or $R_{k, \ell}(z)$. As $M_{k}$ and $N_{k}$ are coprime, we can always find positive integers $a$ and $b$ such that $a M_{k}-b N_{k}=$ 1. Let $a_{k, 1}$ and $b_{k, 1}$ be the smallest integers that satisfy this condition. Define

$$
a_{k, l}=l a_{k, 1}, \quad b_{k, l}=l b_{k, 1} .
$$

Using the polyphase representation and the noble identities [20], we can redraw the $k$ th subbands of the transmitter and receiver, respectively, as those shown in Figures 3(a) and 3(b). Moreover, since $M_{k}$ and $b_{k, 1}$ are coprime, we have ${ }^{2}$

$$
\left\{\left[b_{k, 1}\right]_{M_{k}},\left[b_{k, 2}\right]_{M_{k}}, \ldots,\left[b_{k, M_{k}-1}\right]_{M_{k}}\right\}=\left\{1,2, \ldots, M_{k}-1\right\}
$$

where $[p]_{q}$ represents $p$ modulo $q$, which is a number between 0 and $q-1$. Thus, in Figure 3(a), the signal $x_{k}(n)$ is split into its polyphase components $\left\{x_{k, 0}(n), x_{k, 1}(n), \ldots\right.$, $\left.x_{k, M_{k}-1}(n)\right\}$. Similarly, $\left\{\hat{x}_{k, 0}(n), \hat{x}_{k, 1}(n), \ldots, \hat{x}_{k, M_{k}-1}(n)\right\}$ in Figure $3(\mathrm{~b})$ are the polyphase components of the signal

\footnotetext{
${ }^{2}$ See homework [20, Problem 4.9].
}

$\hat{x}_{k}(n)$. Using these results, we can redraw Figure 2 as Figure 4. The transceiver in Figure 4 has the same structure as that in Figure 1. Since input signals $x_{i, j}(n)$ are also uncorrelated, we can apply the design method developed in previous sections to obtain the optimal $R_{k, \ell}(z)$ and $E_{k, \ell}(z)$. The filters $F_{k}(z)$ and $H_{k}(z)$ can be obtained from (33).

\section{SIMULATIONS}

In this section, we provide two examples to show the performance of nonuniform filter bank transceivers designed by using the proposed method. It is emphasized that in transceiver designs, the nearly zero ISI property is guaranteed by a high SIR value and passband flatness is not needed. We assume that the channel noise $v(n)$ is AWGN in the following examples.

Example 1. In this example, we design nonuniform filter bank transceivers with integer sampling factors. The number of subbands is $M=4$ and the sampling factors are $\left\{N_{0}\right.$, $\left.N_{1}, N_{2}, N_{3}\right\}=\{2,4,8,8\}$. Four-tap channels are used here. A total of 100 randomly generated iid channels are employed in the simulation. We assume that channel impulse responses are known. All the transmitting and receiving filters are of 


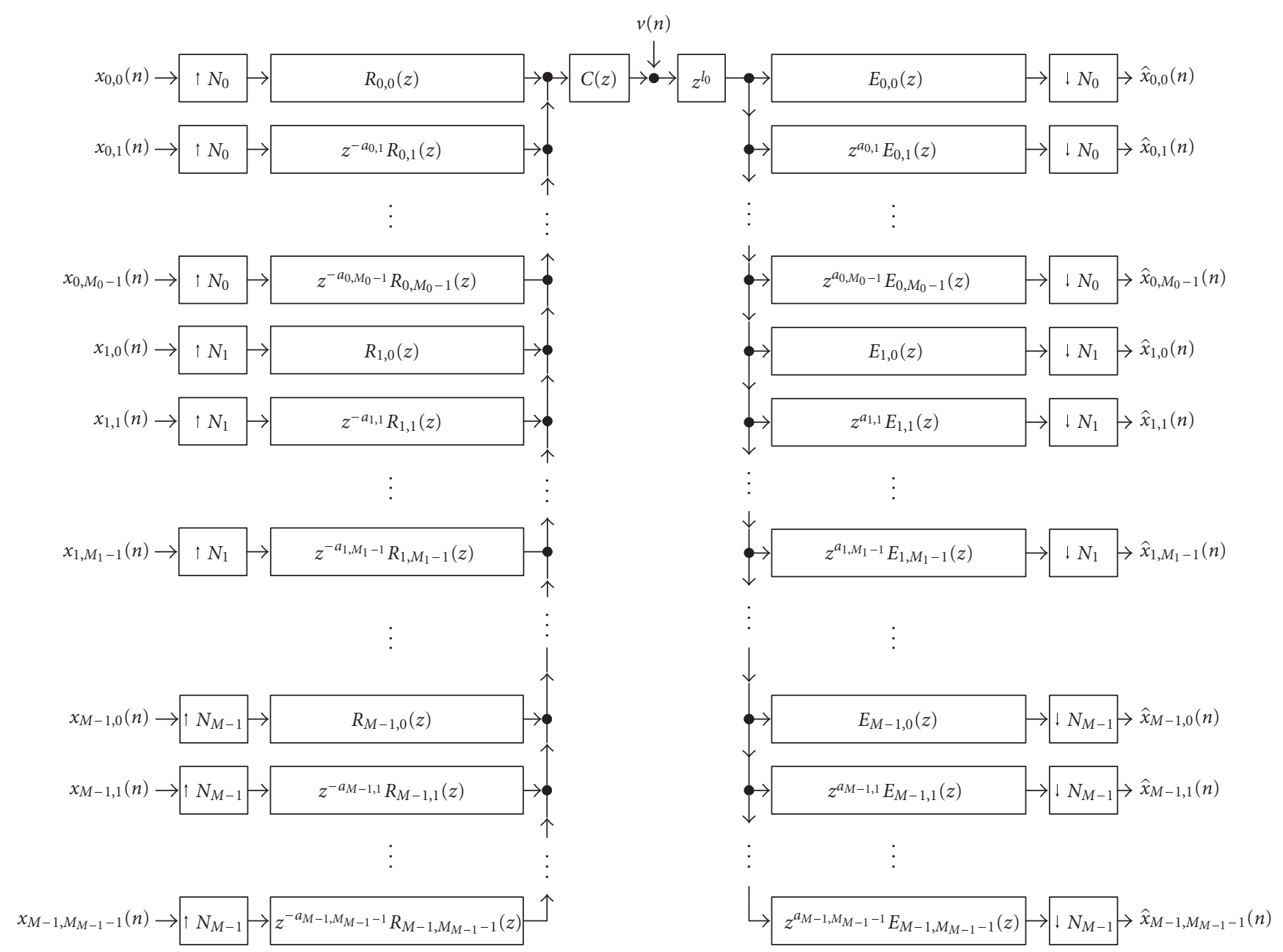

FIGURE 4: Equivalent circuit of the nonuniform filter bank transceiver with rational sampling factors in Figure 2.

order 56. We consider the iterative algorithm for both cases of with and without frequency criteria. For the case with frequency criteria (indicated as fc), the weights for the stopband energy are chosen as $c_{f, 0}=c_{f, 1}=c_{h, 0}=c_{h, 1}=0.05$, and $c_{f, 2}=c_{f, 3}=c_{h, 2}=c_{h, 3}=0.4$. We plot the SIR averaged over the 100 random channels versus the number of iterations and the results are shown in Figure 5. From the figure, we see that the average SIR increases with the number of iterations. When no frequency criteria are applied, the average SIR increases by about $15 \mathrm{~dB}$ and it can be as high as $56 \mathrm{~dB}$ after 400 iterations. Even when the frequency criteria are applied, the average SIR increases by more than $8 \mathrm{~dB}$. Thus the incorporation of frequency criteria results in a loss of SIR by $7 \mathrm{~dB}$. To show the improvement in frequency response when the frequency criteria are applied, we plot the magnitude responses of the transceiver optimized for one particular channel-Channel A after the 200th iteration. The impulse response of Channel $\mathrm{A}$ is given by

$$
\text { Channel A }=\left[\begin{array}{llll}
0.2218 & -0.475 & 0.3906 & 0.2845
\end{array}\right] \text {. }
$$

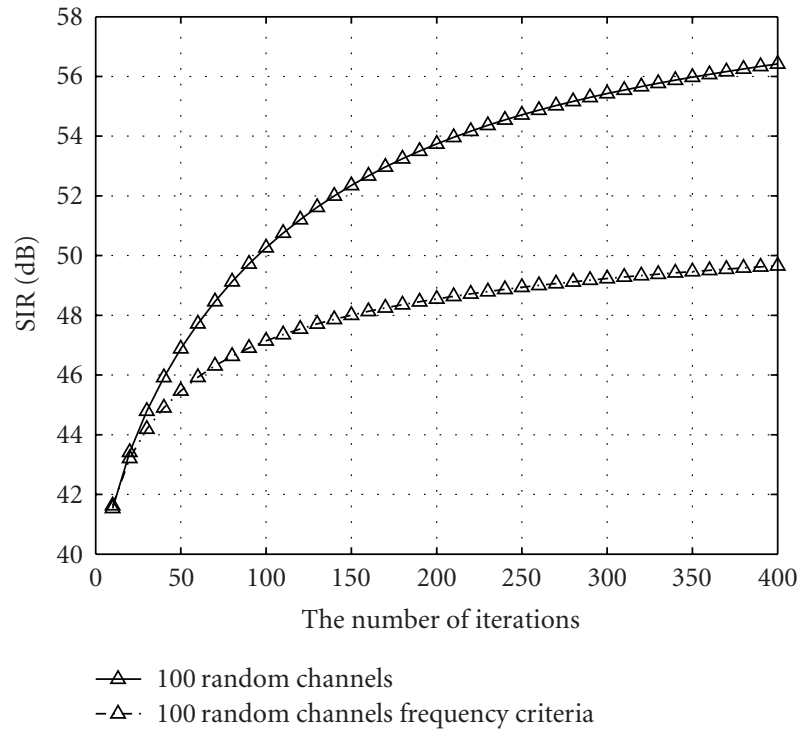

FIGURE 5: SIR versus the number of iterations. 


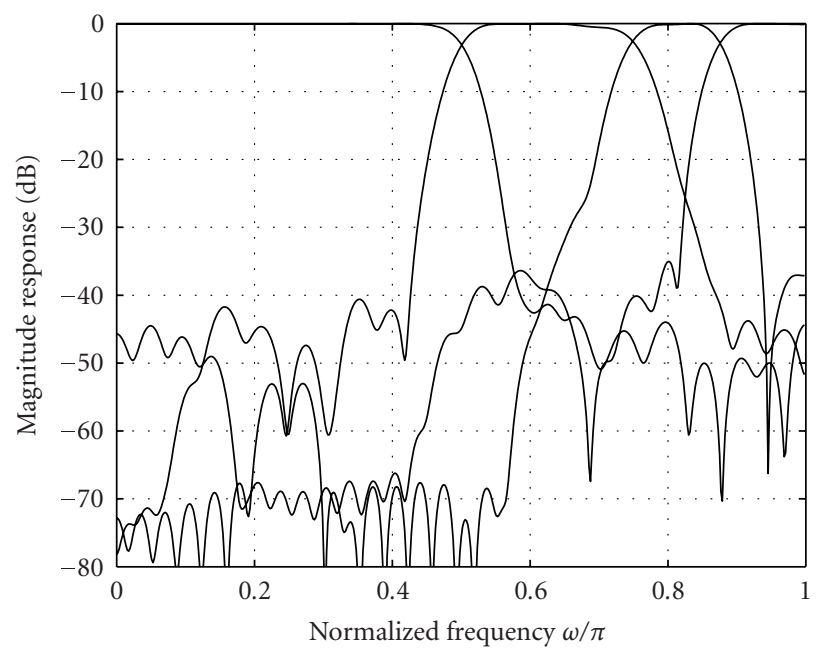

Figure 6: Magnitude responses of the transmitting filters (no frequency criteria).

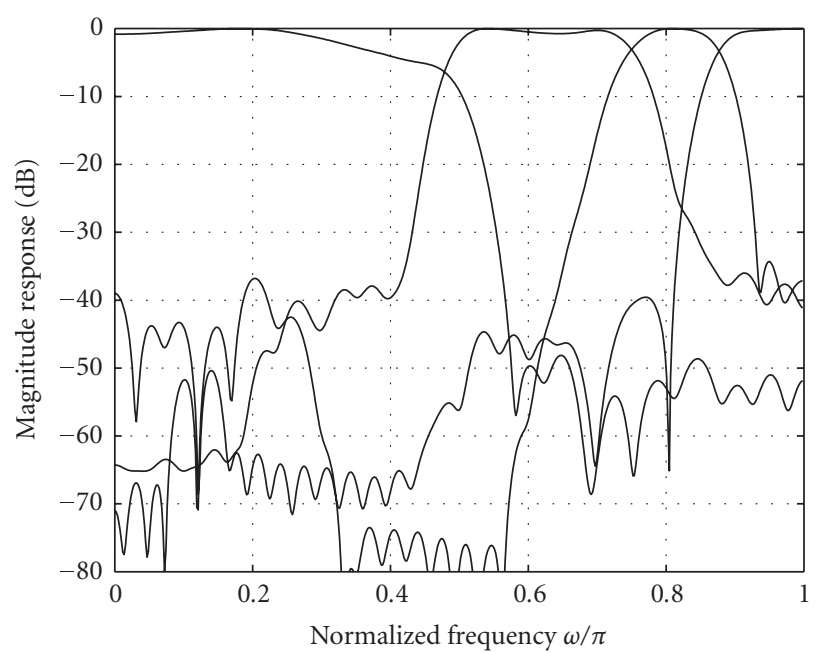

FIGURE 7: Magnitude responses of the receiving filters (no frequency criteria).

The results are shown in Figures 6, 7, 8, and 9. Comparing the results in Figures 6 and 7 with those in Figures 8 and 9, we can see that the incorporation of the frequency criteria improves the frequency characteristics of the transceiver significantly. The tradeoff is a loss in SIR of around $7 \mathrm{~dB}$.

Example 2. In this example, we design two-band nonuniform filter bank transceivers with rational sampling factors, where $N_{0}=N_{1}=5, M_{0}=2$, and $M_{1}=3$. A total of $100 \mathrm{iid}$ channels with 4 taps are randomly generated. The filter orders are $N_{f_{0}}=N_{h_{0}}=58$ and $N_{f_{1}}=N_{h_{1}}=87$. The transmitting filters $F_{0}(z)$ and $F_{1}(z)$ are, respectively, initialized as good lowpass and highpass filters with a passband bandwidth of $2 \pi / 5$. We consider 3 cases: (i) optimization without fre-

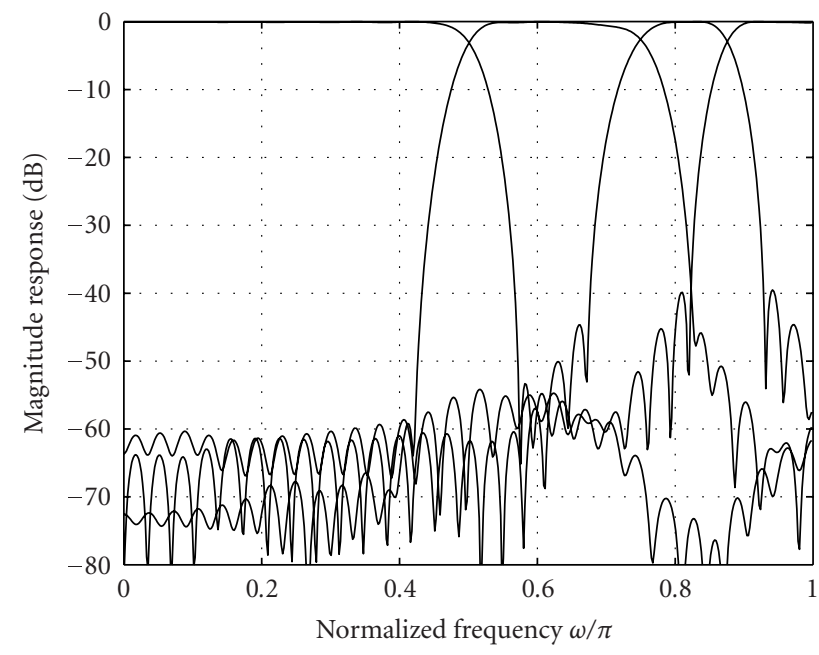

FIGURE 8: Magnitude responses of the transmitting filters (with frequency criteria).

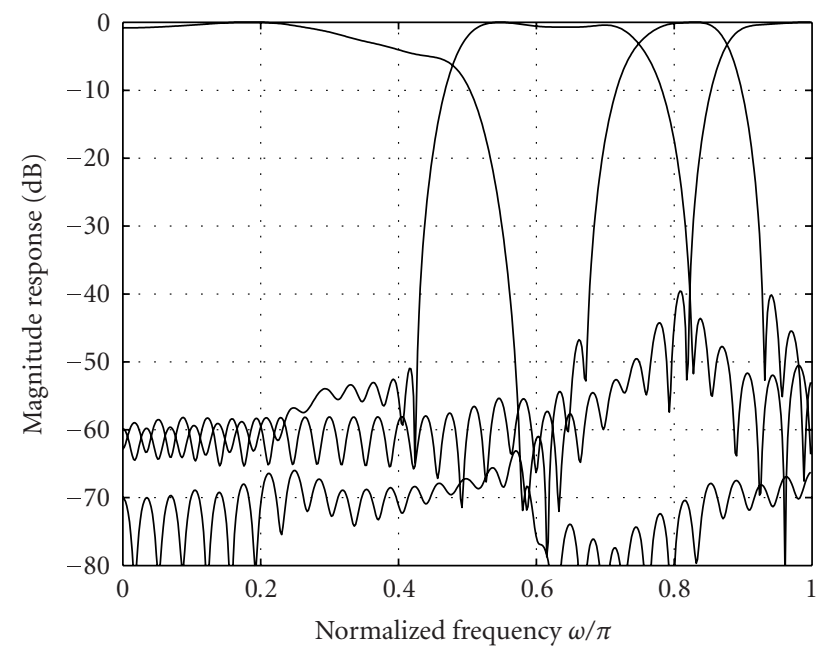

FIGURE 9: Magnitude responses of the transmitting filters (with frequency criteria).

quency criteria (indicated by $c=0$ ); (ii) optimization with frequency criteria and the weights on the stopband energy are $c_{f, 0}=c_{f, 1}=c_{h, 0}=c_{h, 1}=c=0.1$ (indicated by $c=0.1$ ); (iii) optimization with frequency criteria and the weights on the stopband energy are $c_{f, 0}=c_{f, 1}=c_{h, 0}=c_{h, 1}=c=10$ (indicated by $c=10)$. The SIR averaged over 100 random channels versus the number of iterations are given in Figure 10 for the three different values of $c$. From the figure, we see that the SIR is smaller when we impose frequency criteria. The heavier the frequency criteria, the lower the SIR. Comparing the cases of $c=10$ and $c=0$, the loss of SIR (after 200 iterations) is around $6 \mathrm{~dB}$. Even with the frequency weighting of $c=10$, the SIR can be as high as $47 \mathrm{~dB}$, a value that is good enough for many applications. To demonstrate the effect of adding frequency criteria, we plot the filter magnitude responses for 


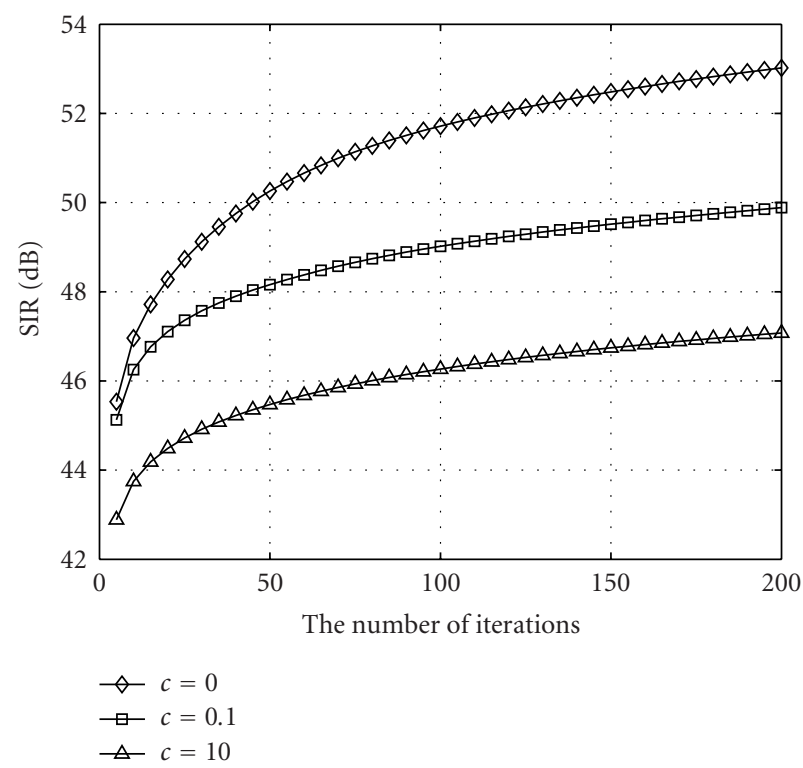

FIGURE 10: SIR versus the number of iterations.

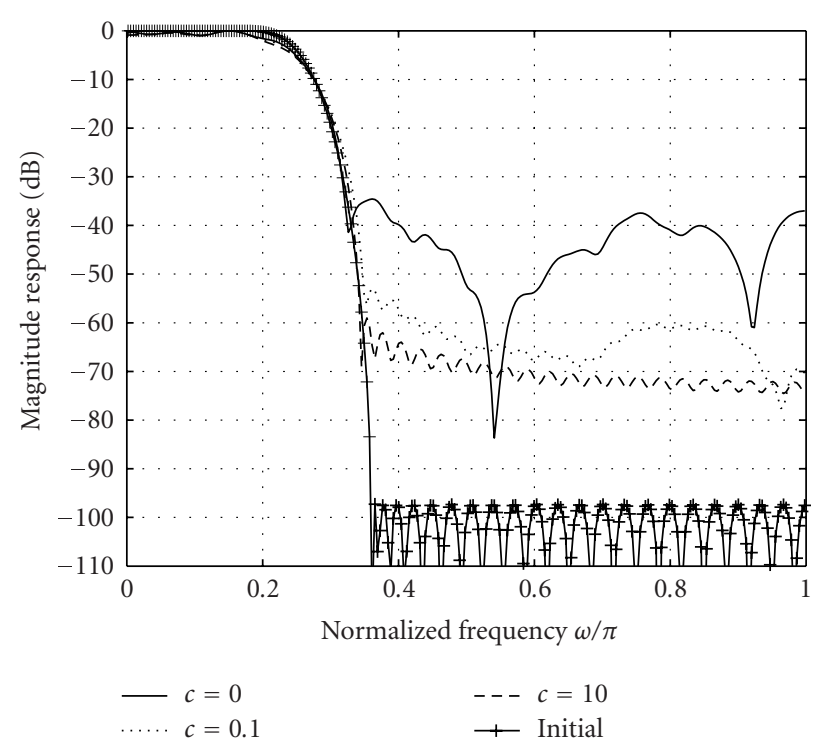

Figure 11: Magnitude response of $F_{0}(z)$.

one particular channel—Channel B after 200 iterations. The impulse response of Channel B is

$$
\text { Channel B }=\left[\begin{array}{llll}
-0.44270 & -0.42492 & 0.39377 & 0.34971
\end{array}\right] \text {. }
$$

The magnitude responses of the initial filters are given in Figures 11 and 12. The results are shown in Figures 11, 12, 13, and 14 (for the purpose of comparison, we also plot the initial $\left|F_{i}\left(e^{j \omega}\right)\right|$ in the same figure). From the figure, it is clear that without any frequency weighting, the magnitude responses degrade significantly after 200 iterations and the

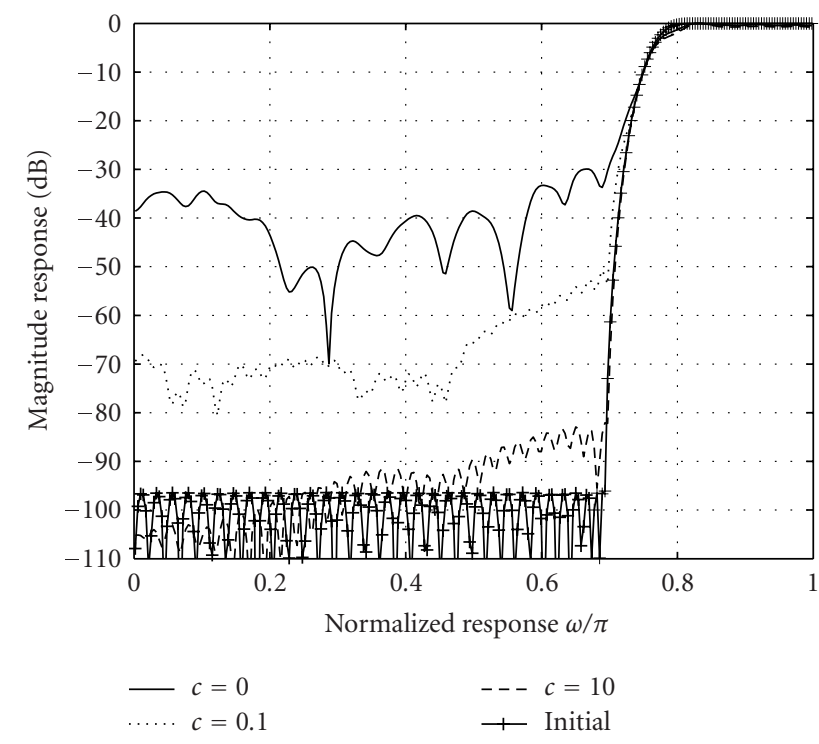

Figure 12: Magnitude response of $F_{1}(z)$.

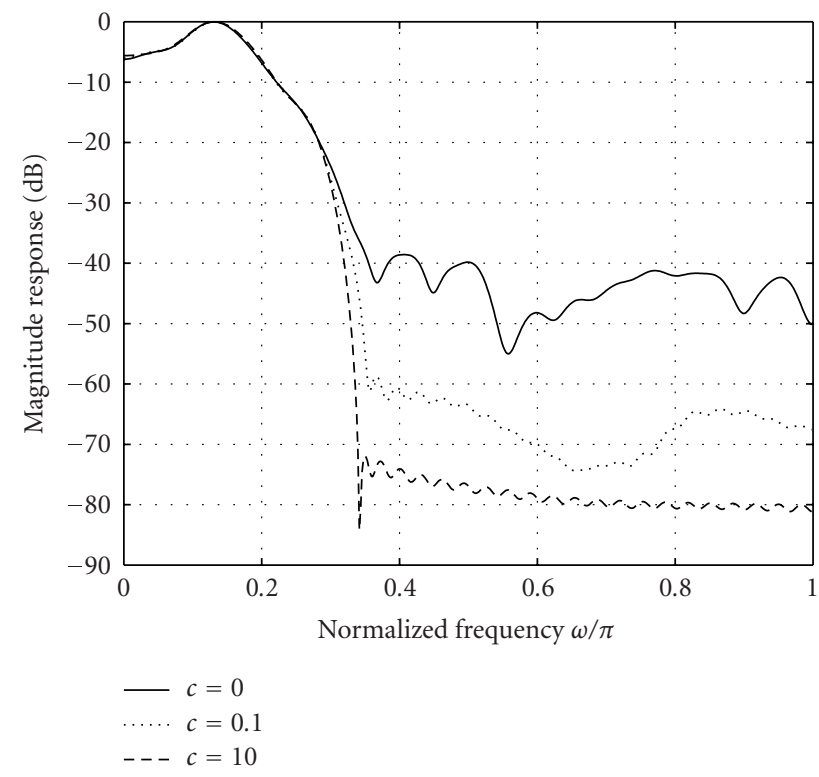

FIgURE 13: Magnitude response of $H_{0}(z)$.

frequency weighting can greatly enhance the selectivity of filters.

\section{CONCLUSION}

In this paper, we propose a method for designing nonuniform filter bank transceivers for frequency selective channels. By expressing the SIR as Rayleigh-Ritz ratios of transmitting and receiving filters respectively, we can iteratively optimize the filters so that SIR is maximized. Moreover, a new cost function that incorporates the filter frequency response is introduced. Iterative optimization algorithm based on the new 


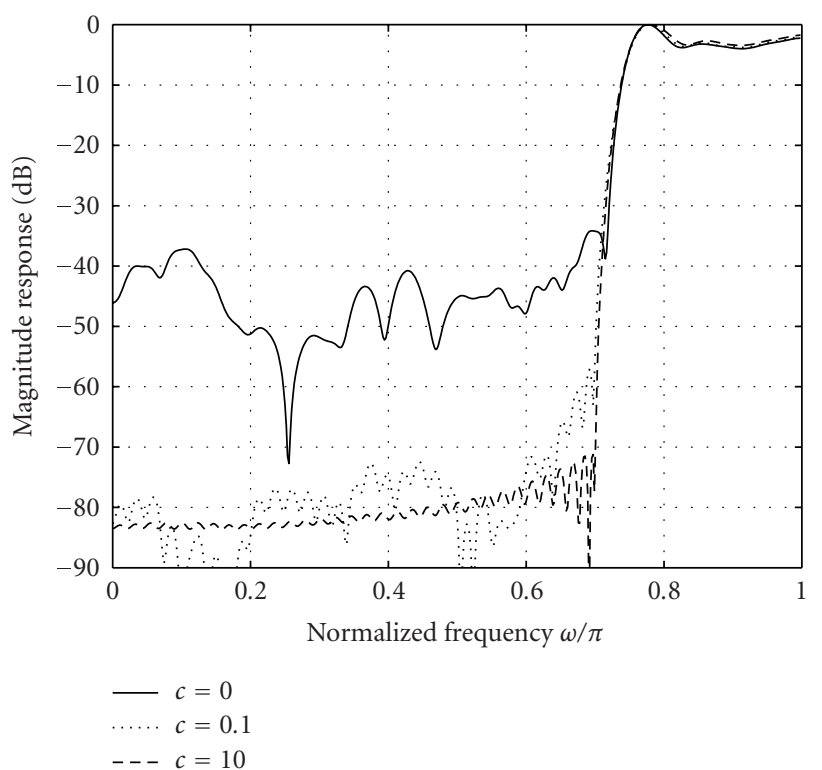

FIGURE 14: Magnitude response of $H_{1}(z)$.

cost function yields transceivers with high SIR as well as good frequency responses.

\section{APPENDIX}

In the following, we will prove that the transceiver in Figure 1 is ISI-free if and only if the transfer function $T_{i, j}(z)$ in (7) satisfies (8). Suppose that $T_{i, j}(z) \neq 0$ for some $i \neq j$. Let $t_{i, j}(k)$ be one of the nonzero coefficients. So $T_{i, j}(z)$ contains the term $t_{i, j}(k) z^{-k}$. Note that the integers $p_{i, j}$ and $p_{j, i}$ are coprime. Thus there exist integers $a$ and $b$ such that $a p_{i, j}+b p_{j, i}=k$. As the inputs $X_{i}(z)$ are arbitrary, let us take $X_{i}(z)=z^{a}$ and $X_{l}(z)=0$ for all $l \neq i$. From (6), one can find that $\hat{X}_{j}(z)$ will contain the term $t_{i, j}(k) z^{-b}$. That means, the $i$ th transmitted signal is causing interference to the $j$ th output of the receiver. Therefore we should have $T_{i, j}(z)=0$ for all $i \neq j$. For the case of $i=j$, it is clear from (6) that the transceiver is ISI-free if and only if $T_{j, j}(z)=G_{j}$ for some constant $G_{j}$.

\section{ACKNOWLEDGMENT}

This work was supported in part by the National Science Council of Taiwan, under Contracts NSC94-2752-E-002006-PAE, NSC94-2213-E-002-075, and NSC94-2213-E-009038.

\section{REFERENCES}

[1] R. Van Nee and R. Prasad, OFDM Wireless Multimedia Communication, Artech House, Boston, Mass, USA, 2000.

[2] T. H. Luo, C. H. Liu, S.-M. Phoong, and Y.-P. Lin, "Design of channel-resilient DFT bank transceivers," in Proceedings of 13th European Signal Processing Conference (EUSIPCO '05), Antalya, Turkey, September 2005.
[3] M. Vetterli, "Perfect transmultiplexers," in Proceedings of IEEE International Conference on Acoustics, Speech and Signal Processing (ICASSP '86), vol. 11, pp. 2567-2570, Tokyo, Japan, April 1986.

[4] S. D. Sandberg and M. A. Tzannes, "Overlapped discrete multitone modulation for high speed copper wire communications," IEEE Journal on Selected Areas in Communications, vol. 13, no. 9, pp. 1571-1585, 1995.

[5] B.-S. Chen, C.-L. Tsai, and Y.-F. Chen, "Mixed $H_{2} / H_{\infty}$ filtering design in multirate transmultiplexer systems: LMI approach," IEEE Transactions on Signal Processing, vol. 49, no. 11, pp. 2693-2701, 2001.

[6] P. Martin-Martin, F. Cruz-Roldan, and T. Saramaki, "Optimized transmultiplexers for multirate systems," in Proceedings of IEEE International Symposium on Circuits and Systems (ISCAS '05), vol. 2, pp. 1106-1109, Kobe, Japan, May 2005.

[7] A. D. Rizos, J. G. Proakis, and T. Q. Nguyen, "Comparison of DFT and cosine modulated filter banks in multicarrier modulation," in Proceedings of IEEE Global Telecommunications Conference (GLOBECOM '94), vol. 2, pp. 687-691, San Francisco, Calif, USA, November-December 1994.

[8] S. Govardhanagiri, T. Karp, P. Heller, and T. Nguyen, "Performance analysis of multicarrier modulation systems using cosine modulated filter banks," in Proceedings of IEEE International Conference on Acoustics, Speech and Signal Processing (ICASSP '99), vol. 3, pp. 1405-1408, Phoenix, Ariz, USA, March 1999.

[9] T. Ihalainen, T. H. Stitz, and M. Renfors, "Efficient per-carrier channel equilier for filter bank based multicarrier systems," in Proceedings of IEEE International Conference on Acoustics, Speech and Signal Processing (ICASSP '05), Philadelphia, Pa, USA, March 2005.

[10] S.-M. Phoong, Y. Chang, and C.-Y. Chen, "DFT-modulated filterbank transceivers for multipath fading channels," IEEE Transactions on Signal Processing, vol. 53, no. 1, pp. 182-192, 2005.

[11] S. Dasgupta and A. Pandharipande, "Optimum multiflow biorthogonal DMT with unequal subchannel assignment," IEEE Transactions on Signal Processing, vol. 53, no. 9, pp. 35723582, 2005.

[12] T. Liu and T. Chen, "Design of multichannel nonuniform transmultiplexers using general building blocks," IEEE Transactions on Signal Processing, vol. 49, no. 1, pp. 91-99, 2001.

[13] P.-Q. Hoang and P. P. Vaidyanathan, "Non-uniform multirate filter banks: theory and design," in Proceedings of IEEE International Symposium on Circuits and Systems, vol. 1, pp. 371-374, Portland, Ore, USA, May 1989.

[14] S. Akkarakaran and P. P. Vaidyanathan, "New results and open problems on nonuniform filter-banks," in Proceedings of IEEE International Conference on Acoustics, Speech, and Signal Processing (ICASSP '99), vol. 3, pp. 1501-1504, Phoenix, Ariz, USA, March 1999.

[15] J. Kovacevic and M. Vetterli, "Perfect reconstruction filter banks with rational sampling factors," IEEE Transactions on Signal Processing, vol. 41, no. 6, pp. 2047-2066, 1993.

[16] J. Princen, "Design of nonuniform modulated filterbanks," IEEE Transactions on Signal Processing, vol. 43, no. 11, pp. 2550-2560, 1995.

[17] K. Nayebi, T. P. Barnwell III, and M. J. T. Smith, "Nonuniform filter banks: a reconstruction and design theory," IEEE Transactions on Signal Processing, vol. 41, no. 3, pp. 1114-1127, 1993.

[18] F. Argenti, B. Brogelli, and E. Del Re, "Design of pseudo-QMF banks with rational sampling factors using several prototype 
filters," IEEE Transactions on Signal Processing, vol. 46, no. 6, pp. 1709-1715, 1998.

[19] C. Y.-F. Ho, B. W.-K. Ling, Y.-Q. Liu, P. K.-S. Tam, and K.-L. Teo, "Optimal design of nonuniform FIR transmultiplexer using semi-infinite programming," IEEE Transactions on Signal Processing, vol. 53, no. 7, pp. 2598-2603, 2005.

[20] P. P. Vaidyanathan, Multirate Systems and Filter Banks, Prentice Hall, Englewood Cliffs, NJ, USA, 1993.

[21] R. A. Horn and C. R. Johnson, Matrix Analysis, Cambridge University Press, Cambridge, UK, 1985.

Han-Ting Chiang was born in Taipei, Taiwan, in 1980. He received the B.S. degree in electrical engineering from the National Tsing Hua University, Hsinchu, Taiwan, and the M.S. degree in electrical engineering from the National Taiwan University, Taipei, Taiwan, in 2003 and 2005, respectively. His research interests include signal processing for communications, wireless communications, and multimedia signal processing.

See-May Phoong was born in Johor, Malaysia, in 1968. He received the B.S. degree in electrical engineering from the National Taiwan University (NTU), Taipei, Taiwan, in 1991 and M.S. and Ph.D. degrees in electrical engineering from the California Institute of Technology (Caltech), Pasadena, Calif, in 1992 and 1996, respectively. He was with the faculty of the Department of Electronic and Electrical Engineer-

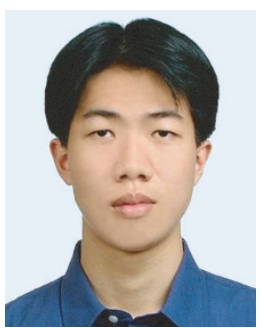
ing, Nanyang Technological University, Singapore, from September 1996 to September 1997. In September 1997, he joined the Graduate Institute of Communication Engineering and the Department of Electrical Engineering, NTU, as an Assistant Professor, and since August 2006, he has been a Professor. He is currently an Associate Editor for the IEEE Transactions on Circuits and Systems I. He has previously served as an Associate Editor for Transactions on Circuits and Systems II: Analog and Digital Signal Processing (January 2002-December 2003) and IEEE Signal Processing Letters (March 2002-February 2005). His interests include multirate signal processing, filter banks and their application to communications. $\mathrm{He}$ received the Charles H. Wilts Prize (1997) for outstanding independent research in electrical engineering at Caltech. He was also a recipient of the Chinese Institute of Electrical Engineerings Outstanding Youth Electrical Engineer Award (2005).

Yuan-Pei Lin was born in Taipei, Taiwan, 1970. She received the B.S. degree in control engineering from the National ChiaoTung University, Taiwan, in 1992, and the M.S. degree and the Ph.D. degree, both in electrical engineering from California Institute of Technology, in 1993 and 1997, respectively. She joined the Department of Electrical and Control Engineering of $\mathrm{Na}$ tional Chiao-Tung University, Taiwan, in

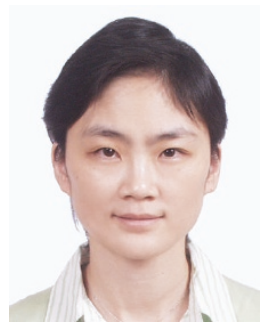

1997. Her research interests include digital signal processing, multirate filter banks, and signal processing for digital communication, particularly the area of multicarrier transmission. She is a recipient of 2004 Ta-You Wu Memorial Award. She served as an Associate
Editor for IEEE Transaction on Signal Processing (2002-2006). She is currently an Associate Editor for IEEE Transaction on Circuits and Systems II, EURASIP Journal on Advances in Signal Processing, and Multidimensional Systems and Signal Processing, Academic Press. She is also a distinguished Lecturer of the IEEE Circuits and Systems Society for 2006-2007. 